\title{
Fiber-coupled displacement interferometry without periodic nonlinearity
}

\author{
Jonathan D. Ellis, ${ }^{1,2, *}$ Arjan J. H. Meskers, ${ }^{1}$ Jo W. Spronck, ${ }^{1}$ and Robert H. Munnig Schmidt ${ }^{1}$ \\ ${ }^{1}$ PME: Mechatronic System Design, Delft University of Technology, Mekelweg 2, 2628 CD, Delft, The Netherlands \\ ${ }^{2}$ Mechanical Engineering Department \& Institute of Optics, University of Rochester, \\ 233 Hopeman Building, Rochester, New York 14627-0132, USA \\ ${ }^{*}$ Corresponding author: j.d.ellis@rochester.edu
}

Received June 20, 2011; revised August 3, 2011; accepted August 18, 2011;

posted August 19, 2011 (Doc. ID 149598); published September 12, 2011

Displacement interferometry is widely used for accurately characterizing nanometer and subnanometer displacements in many applications. In many modern systems, fiber delivery is desired to limit optical alignment and remove heat sources from the system, but fiber delivery can exacerbate common interferometric measurement problems, such as periodic nonlinearity, and account for fiber-induced drift. In this Letter, we describe a novel, general Joo-type interferometer that inherently has an optical reference after any fiber delivery that eliminates fiber-induced drift. This interferometer demonstrated no detectable periodic nonlinearity in both free-space and fiber-delivered variants. (C) 2011 Optical Society of America

OCIS codes: $120.0120,120.2650,120.3180,120.3930,120.4570,120.4820$.

Heterodyne displacement measuring interferometry is a widely applied tool used in gravitational wave detection [1], particle and virus detection [2], characterizing basil membrane mechanics in animal cochlea [3] ], quantifying electrostrictive strains [4]], dilatometry [్], stage metrology [음 $]$ ], and part calibration [10]. Heterodyne interferometry is used because of its high dynamic range, high signal-to-noise ratio, and direct traceability to the meter [11]. The limits to achieving subnanometer performance can be categorized into three error sources: laser frequency stability [11], refractive index fluctuations in noncommon optical paths [12], and periodic nonlinearity in the phase measurement [13-15].

Employing a highly stable laser source [16] and conditioning the measurement environment can mitigate the effects of laser frequency instability and refractive index fluctuations. However, periodic nonlinearity in the measurement is more difficult to eliminate because it arises from a combination of source mixing, manufacturing tolerances, and imperfect alignment. Fiber delivery will inherently decrease polarization stability and add timevarying effects, which further complicates the signal processing for interferometers susceptible to periodic nonlinearity [17].

Several heterodyne interferometer configurations have been developed that limit the chances for periodic nonlinearity [18-23]. These interferometers generally use a spatially separated source and limit all reference and measurement beam overlaps until the final interfering surface prior to detection.

In this Letter, we present a general, fiber-coupled Joo-type interferometer with a novel design based on previous Joo-type retroreflector (RR) and plane mirror target interferometers [21,23], which is more suitable for multiaxis systems and has the same footprint on the measurement target as a typical Michelson plane mirror interferometer. Additionally, we compare the results from periodic error analyses between fiber- and free-space-delivered variants and characterize the nominal drift from fiber-induced Doppler shifts.
Figure 1 shows a schematic of the general Joo-type interferometer. Two spatially separated beams, horizontally polarized with slightly different optical frequencies $\left(f_{1}, f_{2}\right)$ are used as the input. When fiber coupling is employed, the phase of the respective optical frequencies $\left(\theta_{1}, \theta_{2}\right)$, vary in time.

Both beams enter a beamsplitter (BS), where they are split equally. The reflected beams diagonally cross in a large RR due to the RR's point symmetry. They then travel back to the BS, where they interfere with their respective measurement arms.

The initially transmitted beams at the BS also transmit through the polarizing beamsplitter (PBS) and pass through the quarter-wave plate (QWP) oriented at $45^{\circ}$ about the azimuthal angle. The measurement beam reflects from a mirror $\left(\mathrm{M}_{m}\right)$ attached to the moving stage, whereas the reference beam reflects from a mirror attached to the interferometer $\left(\mathrm{M}_{r}\right)$. Upon reflection, both beams pass back through the QWP, becoming vertically polarized where they reflect at the PBS. Each beam is then vertically displaced by its own $R R$ and reflected from the PBS. Both beams pass through the QWP for a third time, reflect from their respective mirrors, and pass through the QWP for a final time. The beams are then horizontally polarized again and pass through the PBS where they interfere with their

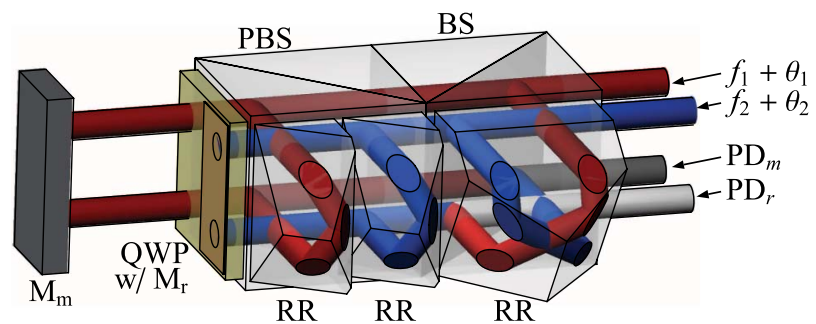

Fig. 1. (Color online) Schematic of the general Joo-type interferometer with the same measurement target footprint as a traditional plane mirror interferometer. The two spatially separated input beams have a slight optical frequency difference and varying phases due to fiber-induced Doppler shifts. No beam overlaps occur until the final interfering surface. 


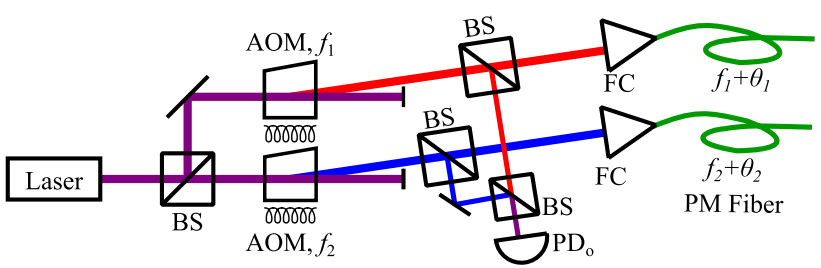

Fig. 2. (Color online) Two AOMs driven at slightly different frequencies frequency modulate an optical beam from a stabilized source. Prior to fiber coupling into PM fibers, parts of each beam are split and combined to generate an optical reference.

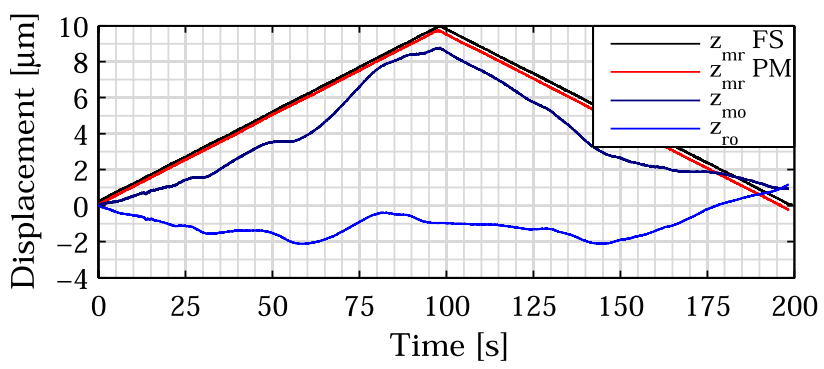

Fig. 3. (Color online) Linear displacement of $10 \mu \mathrm{m}$ comparing the free space (FS) and fiber-coupled (PM) general Joo-type interferometer. Using a reference prior to the fiber delivery causes micrometer-level errors from fiber thermal expansion, while using a reference postfiber eliminates fiber-induced errors. The FS and PM measurements are offset for clarity, and the peak dwell time differs by $1 \mathrm{~s}$.

respective reference arms and are detected with two photodetectors $\left(\mathrm{PD}_{m}, \mathrm{PD}_{r}\right)$.

An optical reference prior to fiber coupling is needed to assess fiber-induced Doppler shifts; see Fig. 2. The optical beam from a frequency-stabilized source is split equally and passed through two acousto-optic modulators (AOMs) driven at slightly different frequencies. A part of the +1 st-order beams from each AOM is sampled, interfered, and detected by $\mathrm{PD}_{o}$ while the main parts are launched into two polarization maintaining (PM) fibers.

The irradiances at the three detectors $\left(\mathrm{PD}_{o}, \mathrm{PD}_{r}\right.$, and $\mathrm{PD}_{m}$, respectively) are

$$
\begin{aligned}
& I_{o} \propto \cos \left(2 \pi f_{1} t\right) \cos \left(2 \pi f_{2} t\right), \\
& I_{r} \propto \cos \left(2 \pi f_{2} t+\theta_{2}+\theta_{r}\right) \cos \left(2 \pi f_{1} t+\theta_{1}+\delta_{1}\right), \\
& I_{m} \propto \cos \left(2 \pi f_{1} t+\theta_{1}+\theta_{m}\right) \cos \left(2 \pi f_{2} t+\theta_{2}+\delta_{2}\right),
\end{aligned}
$$

where $\theta_{r}$ and $\theta_{m}$ are the Doppler shifts of the reference and measurement mirrors, respectively, and $\delta_{1}$ and $\delta_{2}$ are the phase offsets in the reference arms. The measured irradiances simplify to

$$
\begin{aligned}
& I_{o} \propto \cos \left(2 \pi f_{s} t\right), \\
& I_{r} \propto \cos \left(2 \pi f_{s} t+\theta_{1}-\theta_{2}-\theta_{r}+\delta_{1}\right), \\
& I_{m} \propto \cos \left(2 \pi f_{s} t+\theta_{1}-\theta_{2}+\theta_{m}-\delta_{2}\right),
\end{aligned}
$$

after substituting $\left(f_{1}-f_{2}=f_{s}\right)$ and ignoring the optical frequencies too high to detect. Assuming thermal gradients in the interferometer are minimized, $\delta_{1}=\delta_{2}=0$. The three signals are used to generate three difference phase measurements. The measured phase difference between $I_{r}$ and $I_{o}$ is $\phi_{r o}=\Delta \theta_{f}-\theta_{r}$ where $\theta_{f}=\theta_{1}-\theta_{2}$. Similarly, $\phi_{m o}=\Delta \theta_{f}+\theta_{m}$, and $\phi_{m r}=\theta_{m}+\theta_{r}$. Once the phases are determined, the measured displacements, $\boldsymbol{z}_{\text {ro }}$, $z_{m o}$, and $z_{m r}$ can be readily obtained. Clearly, measuring the phase relative to the optical reference prior to fiber coupling includes the differential phase shift from fiber perturbations. However, measuring both the measurement and reference interferometers after the fibers causes the fiber differential phase shift to cancel. Figure 3 shows a linear scan of $10 \mu \mathrm{m}$ forward and backward at $100 \mathrm{~nm} / \mathrm{s}$ comparing the fiber-coupled system results $\left(z_{m r} \mathrm{PM}\right)$ with a similar free-space delivered version $\left(\boldsymbol{z}_{m r}\right.$ FS) and the fluctuations of the individual interferometers while fiber-coupled $\left(z_{r_{0}}\right.$ and $\left.z_{m o}\right)$. When including the fibers, an error on the order of several micrometers occurs, whereas the $z_{m r}$ measurements, both fiber-coupled and free-space, mirror the stage's drive path.
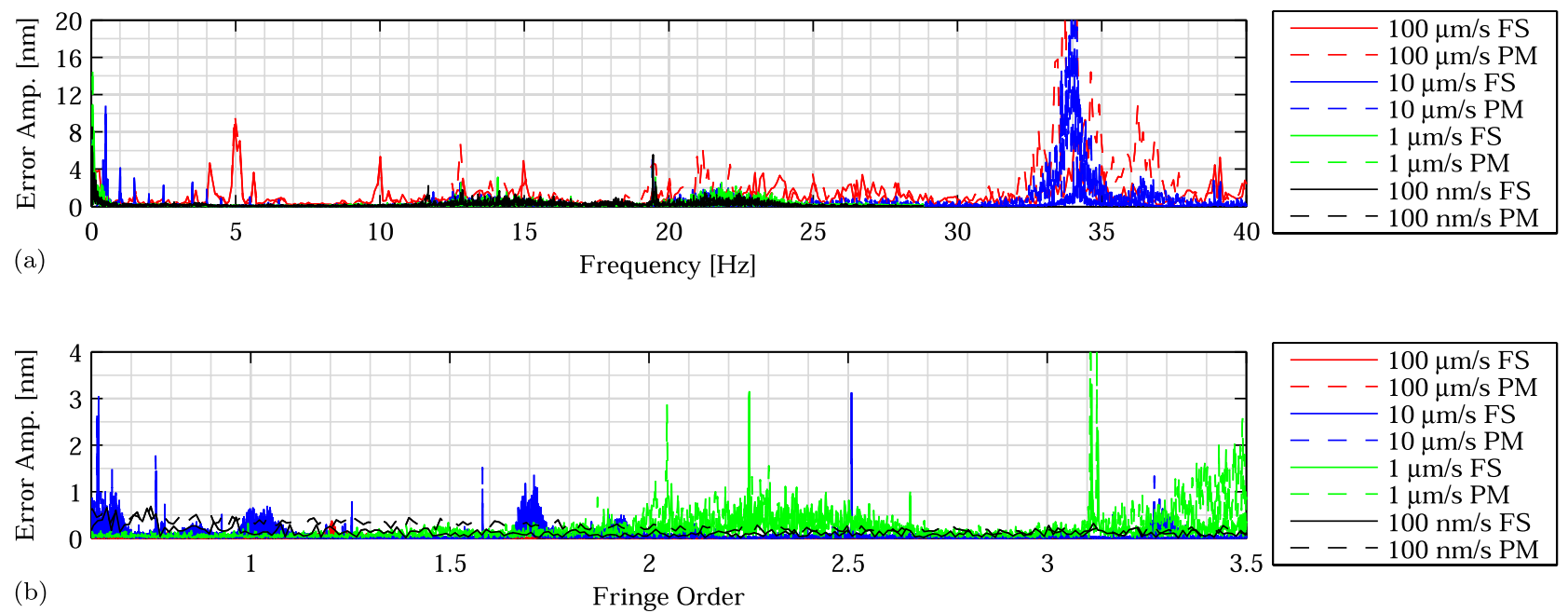

Fig. 4. (Color online) Comparison of displacement errors after detrending for four linear displacements in the (a) frequency and (b) spatial frequency domains. A large error source is at $34 \mathrm{~Hz}$ in (a), which is attributed to the laboratory environment and also apparent in other measurement systems. In the spatial frequency domain, no peaks are observed at the first and second fringe orders, demonstrating no detectable periodic nonlinearity for both the free-space- and fiber-delivered measurements. 
A method for determining periodic nonlinearity is by comparing the error signal in the frequency domain versus the spatial frequency domain (Fig. 4).

In the frequency domain [Fig. 4(a)], a large error source at $34 \mathrm{~Hz}$ is common to all measurements and is attributed to the environmental control and air flow in the laboratory. Additionally, several peaks appear in the $100 \mu \mathrm{m} / \mathrm{s}$ scans from induced vibrations into the optical bench due to a higher scanning velocity. These peaks and locations are apparent in several measurements.

When the measurements are converted to the fringe order in the spatial frequency domain [Fig. 4(b)], no peaks appear in the typical first- and second-order periodic nonlinearity locations. In this case, the first fringe order is $\lambda / N=158 \mathrm{~nm}$ where $\lambda$ is $633 \mathrm{~nm}$ and $N$ is 4 . The second fringe order is half of the first fringe order or $79 \mathrm{~nm}$, and so on. The first and second fringe orders are analyzed because they are the spatial frequency locations concurrent with first- and second-order frequency mixing in heterodyne interferometry [13-15]. The periodic nonlinearity can be distinguished from other error vibrations by changing the stage velocity and sampling parameters [23]. If the peak in the spatial frequency domain shifts, then it is not due to periodic nonlinearity but rather another error source.

In summary, periodic errors can be eliminated from displacement measuring interferometers even with fibercoupled delivery and limited extinction ratios in PM fiber. Two optical beams with slightly different frequencies must be delivered to an interferometer configuration (a general Joo-type in this case) with no overlaps prior to detection. Additionally, an optical reference is needed after the fibers to eliminate fiber-induced errors on the order of micrometers or more.

This work was supported by the Dutch IOP (IPT04001) in the Netherlands. The authors are grateful for the support by Agilent Technologies for providing equipment used during this research.

\section{References}

1. T. Schuldt, M. Gohlke, D. Weise, U. Johann, A. Peters, and C. Braxmaier, Class. Quantum Grav. 26, 085008 (2009).

2. A. Mitra, B. Deutsch, F. Ignatovich, C. Dykes, and L. Novotny, ACS Nano 4, 1305 (2010).

3. N. P. Cooper and W. S. Rhode, Hearing Res. 63, 163 (1992).

4. S. W. P. van Sterkenburg, T. Kwaaitaal, and W. M. M. M. van den Eijnden, Rev. Sci. Instrum. 61, 2318 (1990).

5. D. Ren, K. M. Lawton, and J. A. Miller, Meas. Sci. Technol. 19, 025303 (2008).

6. J. Bryan, D. Carter, and S. Thompson, CIRP Ann. 43, 453 (1994).

7. F. C. Demarest, Meas. Sci. Technol. 9, 1024 (1998).

8. C. Evans, M. Holmes, F. Demarest, D. Newton, and A. Stein, CIRP Ann. 54, 495 (2005).

9. C.-H. Liu, W.-Y. Jywe, C.-C. Hsu, and T.-H. Hsu, Rev. Sci. Instrum. 76, 055110 (2005).

10. P. de Groot, J. Biegen, J. Clark, X. C. de Lega, and D. Grigg, Appl. Opt. 41, 3853 (2002).

11. N. Bobroff, Meas. Sci. Technol. 4, 907 (1993).

12. W. T. Estler, Appl. Opt. 24, 808 (1985).

13. R. C. Quenelle, Hewlett-Packard J. 34, 10 (1983).

14. W. Hou and G. Wilkening, Precis. Eng. 14, 91 (1992).

15. J. M. De Freitas and M. A. Player, J. Mod. Opt. 42, 1875 (1995).

16. J. D. Ellis, K. Joo, E. S. Buice, and J. W. Spronck, Opt. Express 18, 1373 (2010).

17. B. A. W. H. Knarren, S. J. A. G. Cosijns, H. Haitjema, and P. H. J. Schellekens, Precis. Eng. 29, 229 (2005).

18. C. Wu, J. Lawall, and R. D. Deslattes, Appl. Opt. 38, 4089 (1999).

19. J. Lawall and E. Kessler, Rev. Sci. Instrum. 71, 2669 (2000).

20. T. Schmitz and J. Beckwith, J. Mod. Opt. 49, 2105 (2002).

21. K. Joo, J. D. Ellis, J. W. Spronck, P. J. M. van Kan, and R. H. Munnig Schmidt, Opt. Lett. 34, 386 (2009).

22. H. S. Kim, T. Schmitz, and J. Beckwith, J. Opt. Res. 11, $213(2010)$.

23. K. Joo, J. D. Ellis, E. S. Buice, J. W. Spronck, and R. H. Munnig Schmidt, Opt. Express 18, 1159 (2010). 